\title{
Evaluation of the Effect of Radius of Curvature on the Rounded Edge Diffraction Loss Computed by Hacking Method for a Plateau
}

\author{
Swinton C. Nwokonko, Ikechukwu H. Ezeh, Vital K. Onwuzuruike \\ Department of Electrical Engineering, Imo State University (IMSU), Owerri, Nigeria
}

Email address:

ikechukwu4per@yahoo.com (I. H. Ezeh)

To cite this article:

Swinton C. Nwokonko, Ikechukwu H. Ezeh, Vital K. Onwuzuruike. Evaluation of the Effect of Radius of Curvature on the Rounded Edge Diffraction Loss Computed by Hacking Method for a Plateau. American Journal of Software Engineering and Applications.

Vol. 6, No. 2, 2017, pp. 49-55. doi: 10.11648/j.ajsea.20170602.17

Received: January 8, 2017; Accepted: January 18, 2017; Published: June 12, 2017

\begin{abstract}
In this paper, the effect of the radius of curvature on the diffraction loss of rounded edge obstruction is presented. The study is conducted for C-band microwave link with a plateau in its path. The plateau has flat to that spans about $1922 \mathrm{~m}$. Two different approaches are used to determine the radius of curvature of the rounded edged fitted to the plateau top. Among the two methods employed, the ITU-R 526-13 method overestimated the radius (about 12,374,693.37 m) as against 59,031.42 m estimated by the second method at the same C-band frequency of $4 \mathrm{GHz}$. Also, high radius of curvature by the ITU-R 526-13 method gave very high diffraction loss value for the plateau. Furthermore, with the ITU-R 526-13 method, the radius of curvature does increase with increase in frequency. In all, the results indicate that the ITU-R 526-13 method is not particularly suitable for estimating the radius of curvature for the rounded edge when applied to a plateau. In addition, a more accurate method is required to estimate the radius of curvature for computing rounded edge diffraction loss.
\end{abstract}

Keywords: Rounded Edge Diffraction, Diffraction Loss, Elevation Profile, Diffraction Parameter, Knife Edge Diffraction, Hacking Rounded Edge Diffraction Method

\section{Introduction}

Most obstacle encountered real world cannot be well modeled by a single knife-edge obstruction approach [1]. As such, the rounded-surface diffraction model, where the diffraction is treated as a broadside cylinder is used in some cases such as hills [1-5]. In this case, the diffraction from a rounded edge is determined by first computing the single knife-edge diffraction and then computing the excess diffraction loss due to the rounded surface. In order to determine the diffraction loss due to the rounded surface, the first step is to determine the radius of a circle fitted to the apex of the obstruction. Then the diffraction loss due to the rounded surface is added to the single knife-edge diffraction loss and the sum gives the total or effective diffraction loss due to the obstruction [1]. Essentially, diffraction losses due to rounded obstacles exceed that over knife edge obstacle [6-9].

There are different methods that have been developed for estimating diffraction over rounded edge. In this paper, the presented by Hacking is used $[2,13]$. In all the rounded edge diffraction methods, the radius of curvature of the rounded edge fitted to the vertex of the obstruction plays significant role in the value of the diffraction loss obtained [2]. So, the focus of this paper is to evaluate the effect of the radius of curvature of the rounded edge on the effective diffraction loss as computed by the Hacking method.

\section{Theoretical Background}

\subsection{The Rounded Plateau Obstruction Geometry and Parameters}

The elevation profile of the plateau obstruction is shown in Figure 1. In this paper, the focus is to evaluate the effect of the radius of curvature on the diffraction loss computed using Hacking method. Two methods are used to estimate the radius of curvature for the rounded edge fitted at the top of the plateau. Particularly, the ITU method gives a range of values for the radius of curvature [14]. As such, different 
diffraction loss values can be obtained. The impact of the radius of curvature of the rounded edge on the diffraction loss is examined.

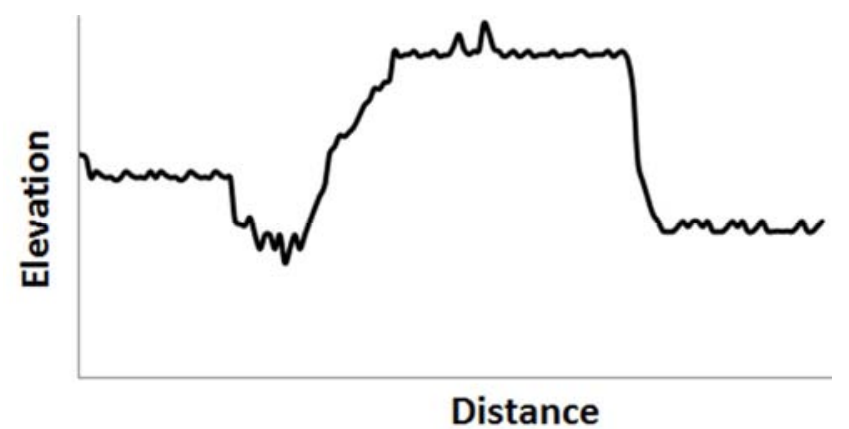

Figure 1. The elevation profile of a plateau as the obstruction in the signal path.

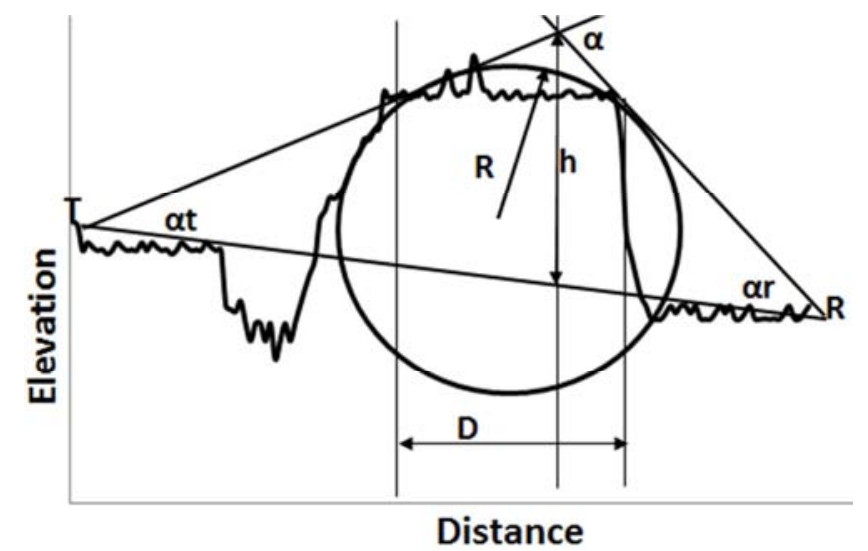

Figure 2. The elevation profile of the plateau obstruction with tangential lines and fitted circle at the vicinity of.

The Plateau Vertex.

The key parameters needed in Hacking method to compute the round edge diffraction loss for the plateau are shown in figure 2. The key dimensions include:

i. S1: the length tangent line $t$ which is the tangent from the transmitter to the path profile.

ii. S2: the length tangent line $r$ which is the tangent from the receiver to the path profile.

Note: S1 is measured from the transmitter to the point where tangent line $t$ intersect tangent line r. Similarly, S2 is measured from the receiver to the point where tangent line $r$ intersect tangent line $t$. In this paper, the tangent line $t$, the tangent line $r$, their tangent points and their lengths denoted as $\mathrm{S} 1$ and $\mathrm{S} 2$ are determined by drawing the tangent line $\mathrm{t}$ and tangent line $r$ on the graphical plot of the path profile. Also, the length of the line of sight (LOS) denoted as S3 is measured out from the graph plot of the path profile.

iii. S3: the length of the line of sight (LOS) which is the line from the transmitter to the receiver.

iv. $\beta$ : The LOS is inclined at angle $\beta$ to the horizontal. The angle which the LOS makes with the horizontal is denoted as $\beta$ where;

$$
\beta=\tan ^{-1}\left(\frac{\mathrm{H}_{\text {elt }}-\mathrm{H}_{\text {elr }}}{\mathrm{d}}\right)
$$

where

$\mathrm{H}_{\text {elt }}$ is the elevation of the transmitter and $\mathrm{H}_{\text {elr }}$ is the elevation of the receiver. $\mathrm{H}_{\text {elt }}$ and $\mathrm{H}_{\mathrm{elr}}$ are obtained from the path profile data.

v. d1: the horizontal distance from the transmitter to the intersection point of the two tangents

vi. d2: the horizontal distance from the receiver to the intersection point of the two tangents

vii. $d$ is the path length, that is the distance between the transmitter and the receiver, and $\mathrm{d}$ is given as;

$$
\mathrm{d}=\mathrm{d}_{\mathbf{t}}+\mathrm{d}_{\mathbf{r}}
$$

$d_{t}$ and $d_{r}$ are measured from the graph plot and the intersection point of tangent line $t$ and tangent line $r$

viii. D: the occultation distance, which is the distance between the tangent point before and the tangent point after the intersection point of tangent line $t$ and tangent line $\mathrm{t}$. $\mathrm{D}$ is the distance between the tangent point of tangent line $t$ with the path profile and the tangent point of tangent line $r$ with the path profile. In this paper, the tangent points are determined by drawing the tangent line $t$ and tangent line $r$ on the graphical plot of the path profile. Then, D is measured from the graph plot as the distance between the tangent points of tangent line $t$ and tangent line $r$.

ix. $\alpha t$ : the angle between the LOS and (tangent line t) the tangent line drawn from the transmitter to the elevation profile.

x. $\alpha$ : the angle between the LOS and (tangent line $r$ ) the tangent line drawn from the receiver to the elevation profile.

The angles $\alpha \mathrm{t}$ and $\alpha \mathrm{r}$ are obtain by cosine rule as follows;

$$
\begin{gathered}
\operatorname{Cos}(\alpha \mathrm{t})=\frac{(\mathrm{S} 1)^{2}+(\mathrm{S} 3)^{2}-(\mathrm{S} 2)^{2}}{2(S 1)(S 3)} \\
\alpha \mathrm{t}=\operatorname{Cos}^{-1}\left(\frac{(\mathrm{S} 1)^{2}+(\mathrm{S} 3)^{2}-(\mathrm{S} 2)^{2}}{2(S 1)(S 3)}\right)
\end{gathered}
$$

Similarly,

$$
\alpha \mathrm{r}=\operatorname{Cos}^{-1}\left(\frac{(\mathrm{S} 2)^{2}+(\mathrm{S} 3)^{2}-(\mathrm{S} 1)^{2}}{2(\mathrm{~S} 2)(S 3)}\right)
$$

The angle $\alpha$ is given as;

$$
\alpha=\alpha \mathrm{t}+\alpha \mathrm{r}
$$

xi. $h$ is the height of the intersection point of tangent line $t$ and tangent line $r$ above the LOS. $h$ is the height of the intersection point of tangent line $t$ and tangent line $r$ above the LOS. $\mathrm{h}$ is given as;

$$
h=\frac{\operatorname{S1}[\operatorname{Sin}(\alpha t)]}{\operatorname{Sin}(90-\beta)}
$$

xii. $\mathrm{v}$ is the diffraction parameter which is given as;

$$
\mathrm{v}=h \sqrt{\frac{2(d 1+d 2)}{\lambda(\mathrm{d} 1)(\mathrm{d} 2)}}
$$

xiii. $\mathrm{R}$ is the radius of the circle fitted to the plateau in the vicinity of the plateau vertex. The radius of the circle 
fitted in the vicinity of the hill vertex. The circle fitted in the vicinity of the hill vertex is tangential to the tangent line $t$ and tangent line $r$. The approximate value of $R$ is estimated from the path profile using the formula [15, $16]$;

$$
R=\frac{2(\mathrm{D})(\mathrm{d} 1)(\mathrm{d} 2)}{(\alpha)\left[\left(\mathrm{d} 1^{2}\right)+\left(\mathrm{d} 2^{2}\right)\right]}
$$

According to ITU-R 526-13 [14], the obstacle radius of curvature corresponds to the radius of curvature of a parabola fitted at the apex of the obstacle profile. Let $r i$ be the radius of curvature corresponding to the sample $i$ of the vertical profile of the ridge.

$$
r_{i}=\frac{x_{i}^{2}}{2\left(y_{i}\right)}
$$

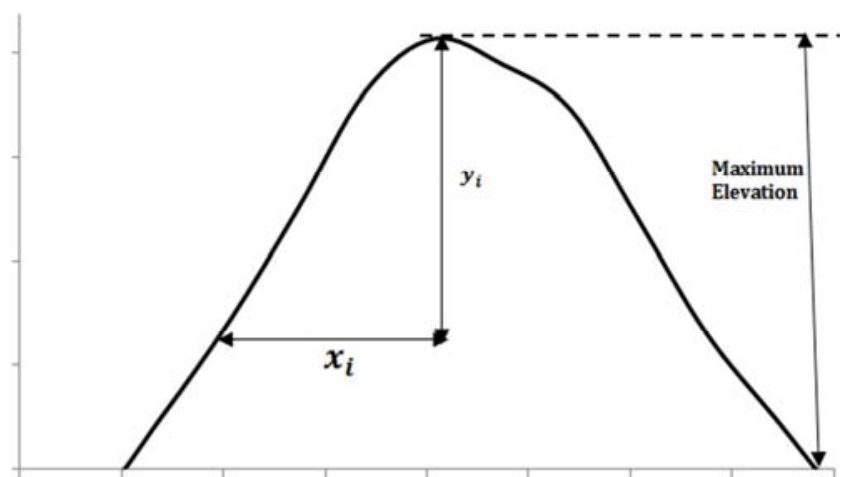

Figure 3. The Geometry of the Vertical Profile of the obstruction used for the determination of the radius of the rounded edge fitted to the vicinity of the obstruction vertex according to ITU Method [14].

$$
\mathrm{A}_{k e}(d B)=\left\{\begin{array}{c}
0 \text { for } \mathrm{v}<-1 \\
20 \log (0.5-0.62 \mathrm{v}) \text { for }-1 \leq \mathrm{v} \leq 0 \\
20 \log (0.5 \exp (-0.95 \mathrm{v})) \text { for } 0 \leq \mathrm{v} \leq 1 \\
20 \log \left(0.4-\sqrt{0.1184-(0.38-0.1 \mathrm{v})^{2}}\right) \text { for } 1 \leq \mathrm{v} \leq 2.4 \\
20 \log \left(\frac{0.225}{v}\right) \text { for } \mathrm{v}>2.4
\end{array}\right\}
$$

where

- $\mathrm{v}$ is the diffraction parameter as given in Eq 8 .

- $\mathrm{h}$ is the line of sight (LOS) clearance height, $\mathrm{h}$ is in meters;

- $d_{t}$ is the distance from the transmitter and $d_{r}$ is the distance from the receiver. $d_{t}$ and $d_{r}$ are is $\mathrm{km}$.

The excess diffraction loss according to Hacking is given as $[2,13]$ :

$$
A_{e x}=11.7(\alpha)\left(\frac{(\pi) R}{\lambda}\right)^{1 / 3}
$$

Where

- $\alpha$ is the exterior angle (in radian) between the tangent line drawn from the transmitter (referred to as tangent line $\mathrm{t})$ and the tangent lines drawn from the receiver (referred to as tangent line $\mathrm{r}$ ). $\alpha$ is given from $\mathrm{Eq} 6, \mathrm{Eq}$ 3 and Eq 5.

- $\mathrm{R}$ is the radius of the circle fitted in the vicinity of the
When fitting the parabola, the maximum vertical distance from the apex to be used in this procedure should be of the order of the first Fresnel zone radius where the obstacle is located. As such, in figure 3, the maximum $y_{i}$ is less or equal to the radius of first Fresnel zone at the point of maximum elevation. In the case of $N$ samples, the median radius of curvature of the obstacle is denoted as $\mathrm{R}$ where [14]:

$$
R=\sum_{i=1}^{i=N}\left(r_{i}\right)=\sum_{i=1}^{i=N}\left(\frac{x_{i}{ }^{2}}{2\left(y_{i}\right)}\right)
$$

\subsection{Hacking Method for Computing Diffraction Loss over Rounded Edge}

Hacking method uses three components to compute the total diffraction loss, $\mathrm{A}_{d B}(v)$ for rounded edge as follows [2, $15,16]$;

$$
\mathrm{A}_{d B}(v)=\mathrm{A}_{k e}+A_{e x}
$$

Where

$\mathrm{A}_{k e}$ is the knife edge diffraction loss $\mathrm{v}$ is the diffraction parameter as given in Eq 8 .

$A_{e x}$ is the excess diffraction loss which is added to the knife edge diffraction loss to account for the rounded hilltop.

The knife edge diffraction loss, $\mathrm{A}_{k e}$ can be determined by Lee's piecewise knife edge diffraction loss approximation model $[17,18]$ expressed in respect of diffraction parameter, $\mathrm{v}$ as follows: obstruction vertex. $\mathrm{R}$ is given in $\mathrm{Eq} 9$.

- $\Lambda$ is the signal wavelength which is given as;

$$
\Lambda=\frac{c}{f}
$$

$\mathrm{f}$ is the frequency in $\mathrm{Hz}$ and $\mathrm{c}$ is the speed of light which is $3 \times 10^{8} \mathrm{~m} / \mathrm{s}$.

\section{Results and Discussions}

The study if conducted for the $\mathrm{C}$-band microwave frequency which ranges from $4 \mathrm{GHz}$ to $8 \mathrm{GHz}$. Specifically, the $4 \mathrm{GHz}, 6 \mathrm{GHz}$ and $8 \mathrm{GHz}$ frequencies are considered in this paper. Table 1 shows the elevation profile data used for the study. According to the data in Table 1, the elevation maximum elevation is $280.93 \mathrm{~m}$ and it occurred at a distance of $4429.74 \mathrm{~m}$ from the transmitter. 
Table 1. The Elevation Profile For The Plateau.

\begin{tabular}{|c|c|c|c|c|c|c|c|c|c|}
\hline Distance (m) & Elevation (m) & Distance (m) & Elevation (m) & Distance (m) & Elevation (m) & Distance (m) & Elevation (m) & Distance (m) & Elevation (m) \\
\hline 0.0 & 262.3 & 1253.7 & 247.7 & 2507.4 & 274.0 & 3761.1 & 280.9 & 5014.8 & 254.4 \\
\hline 41.8 & 261.7 & 1295.5 & 247.2 & 2549.2 & 275.3 & 3802.9 & 279.9 & 5056.6 & 255.7 \\
\hline 83.6 & 247.0 & 1337.3 & 246.7 & 2591.0 & 275.6 & 3844.7 & 280.2 & 5098.4 & 257.0 \\
\hline 125.4 & 253.5 & 1379.1 & 246.4 & 2632.8 & 280.9 & 3886.5 & 280.2 & 5140.2 & 256.6 \\
\hline 167.2 & 255.3 & 1420.9 & 240.9 & 2674.6 & 279.9 & 3928.3 & 280.3 & 5182.0 & 256.1 \\
\hline 209.0 & 247.0 & 1462.7 & 247.7 & 2716.4 & 280.2 & 3970.1 & 280.9 & 5223.8 & 254.0 \\
\hline 250.7 & 253.5 & 1504.4 & 245.2 & 2758.1 & 280.3 & 4011.8 & 279.9 & 5265.5 & 251.1 \\
\hline 292.5 & 254.6 & 1546.2 & 247.7 & 2799.9 & 280.9 & 4053.6 & 280.2 & 5307.3 & 248.0 \\
\hline 334.3 & 245.8 & 1588.0 & 247.7 & 2841.7 & 279.9 & 4095.4 & 280.2 & 5349.1 & 244.4 \\
\hline 376.1 & 239.9 & 1629.8 & 245.2 & 2883.5 & 280.2 & 4137.2 & 280.3 & 5390.9 & 241.0 \\
\hline 417.9 & 235.4 & 1671.6 & 247.7 & 2925.3 & 280.3 & 4179.0 & 280.9 & 5432.7 & 238.5 \\
\hline 459.7 & 231.6 & 1713.4 & 242.6 & 2967.1 & 280.9 & 4220.8 & 280.9 & 5474.5 & 236.0 \\
\hline 501.5 & 232.7 & 1755.2 & 245.2 & 3008.9 & 279.9 & 4262.6 & 280.1 & 5516.3 & 237.4 \\
\hline 543.3 & 236.9 & 1797.0 & 247.7 & 3050.7 & 280.2 & 4304.4 & 280.2 & 5558.1 & 239.9 \\
\hline 585.1 & 241.5 & 1838.8 & 245.2 & 3092.5 & 280.3 & 4346.2 & 280.2 & 5599.9 & 242.5 \\
\hline 626.9 & 247.7 & 1880.6 & 247.7 & 3134.3 & 280.9 & 4388.0 & 280.3 & 5641.7 & 245.6 \\
\hline 668.6 & 245.2 & 1922.3 & 250.2 & 3176.0 & 279.9 & 4429.7 & 280.9 & 5683.4 & 248.4 \\
\hline 710.4 & 231.6 & 1964.1 & 252.6 & 3217.8 & 280.2 & 4471.5 & 279.9 & 5725.2 & 248.3 \\
\hline 752.2 & 232.7 & 2005.9 & 254.8 & 3259.6 & 280.3 & 4513.3 & 280.5 & 5767.0 & 248.2 \\
\hline 794.0 & 231.6 & 2047.7 & 256.7 & 3301.4 & 280.9 & 4555.1 & 280.8 & 5808.8 & 245.2 \\
\hline 835.8 & 232.7 & 2089.5 & 262.5 & 3343.2 & 280.9 & 4596.9 & 279.2 & 5850.6 & 241.7 \\
\hline 877.6 & 236.9 & 2131.3 & 263.7 & 3385.0 & 279.9 & 4638.7 & 273.8 & 5892.4 & 238.8 \\
\hline 919.4 & 241.5 & 2173.1 & 265.7 & 3426.8 & 280.2 & 4680.5 & 260.5 & 5934.2 & 236.6 \\
\hline 961.2 & 247.7 & 2214.9 & 265.5 & 3468.6 & 280.3 & 4722.3 & 257.3 & 5976.0 & 235.1 \\
\hline 1003.0 & 245.2 & 2256.7 & 266.4 & 3510.4 & 280.9 & 4764.1 & 254.3 & 6017.8 & 235.8 \\
\hline 1044.8 & 232.7 & 2298.5 & 267.4 & 3552.2 & 279.9 & 4805.9 & 251.5 & 6059.6 & 236.3 \\
\hline 1086.5 & 236.9 & 2340.2 & 269.3 & 3593.9 & 280.3 & 4847.6 & 249.5 & 6101.3 & 237.1 \\
\hline 1128.3 & 241.5 & 2382.0 & 271.2 & 3635.7 & 280.9 & 4889.4 & 247.4 & 6143.1 & 237.8 \\
\hline 1170.1 & 247.7 & 2423.8 & 272.1 & 3677.5 & 279.9 & 4931.2 & 248.7 & 6184.9 & 238.8 \\
\hline 1211.9 & 245.2 & 2465.6 & 274.2 & 3719.3 & 280.3 & 4973.0 & 251.9 & 6226.7 & 240.2 \\
\hline
\end{tabular}

Table 2. Rounded Edge Parameters For The Plateau Obstruction.

\begin{tabular}{|c|c|c|}
\hline f (GHz) & Frequency & 6000 \\
\hline$\lambda(\mathrm{m})$ & Wavelength & 0.05 \\
\hline $\mathrm{S} 1(\mathrm{~m})$ & The length of the tangent from the transmitter to the intersection point of the two tangent & 4084.652326 \\
\hline $\mathrm{S} 2(\mathrm{~m})$ & the length of the tangent from the receiver to the intersection point of the two tangents & 2142.842069 \\
\hline $\mathrm{S} 3(\mathrm{~m})$ & the length of the tangent from the receiver from the transmitter & 6226.749177 \\
\hline d1 (m) & the distance from the transmitter to the intersection point of the two tangents, that is point & 4084.532567 \\
\hline $\mathrm{d} 2(\mathrm{~m})$ & the distance from the receiver to the intersection point of the two tangents & 2142.177433 \\
\hline $\mathrm{d}(\mathrm{m})$ & the distance from the transmitter to the receiver & 6226.71 \\
\hline$\alpha \mathrm{r}$ (radian) & The angle the tangent line from the receiver makes with the LOS & 0.011204902 \\
\hline$\alpha$ (radian) & Sum of angles $\alpha$ and $\alpha \mathrm{r}$ & 0.03256469 \\
\hline$\beta \alpha($ radian $)$ & The angle the LOS makes with the horizontal & 0.0035473 \\
\hline $\mathrm{h}(\mathrm{m})$ & The LOS clearance height & 45.76746032 \\
\hline $\mathrm{D}(\mathrm{m})$ & the occultation distance & 1922.34 \\
\hline $\mathrm{V}$ & Diffraction parameter for all the methods except ITU-R method & 7.721776507 \\
\hline $\mathrm{V}$ & Diffraction parameter for the ITU-R P.526-13 Method & 0.244008138 \\
\hline $\mathrm{R} 1(\mathrm{~m})=$ & The radius of the circle fitted in the vicinity of the hill vertex & 48561.93427 \\
\hline
\end{tabular}

Table 2 shows the values obtained for the key parameters of the rounded edge plateau obstruction. From Table 2, the path length (d) is $6226.71 \mathrm{~m}$. Also, the tangent from the transmitter and the tangent from the receiver intersected at a distance of $4084.532567 \mathrm{~m}$ from the transmitter and a distance of $2142.177433 \mathrm{~m}$ from the receiver. The line of sight makes an angle of 0.0035473 radians with the horizontal. The LOS clearance height is $45.76746032 \mathrm{~m}$. The occultation distance is $1922.34 \mathrm{~m}$.

Table 3 shows the rounded edge radius computed by Hacking method and by ITU-R 526-13 method. The results in
Table 3 shows that the rounded edge radius computed by Hacking method is not affected by frequency. However, the rounded edge radius computed by ITU-R 526-13 method increases with frequency, as shown in figure 4 . Also, the table 3 shows that the radius computed by the ITU-R 526-13 method exceed that computed by Hacking method by over $20,862 \%$. The difference in radius also increases with increase in frequency.

Table 4 shows the diffraction loss over the rounded edge for different frequencies in the C-band. At each frequency, the diffraction loss is determined using the rounded edge radius 
computed by Hacking method and also using the rounded edge radius computed by ITU-R 526-13 method. The results in Table 4 show that the diffraction loss determined using the rounded edge radius computed by ITU-R 526-13 method is much higher than that obtained using the rounded edge radius computed by
Hacking method. The percentage difference in diffraction loss in the two cases ranges from about $316 \%$ at $4 \mathrm{GHz}$ to about $337 \%$ at $8 \mathrm{GHz}$. In all, the ITU method for determining the rounded edge radius overestimate the value, especially for plateau with flat top that spans relatively large distance.

Table 3. Comparison Of The Effect Of Frequency On The Rounded Edge Radius Computed By Hacking Method and By ITU-R 526-13 Method.

\begin{tabular}{llll}
\hline Frequency (GHz) & $\begin{array}{l}\text { Rounded Edge Radius (m) Computed } \\
\text { By Hacking Method }\end{array}$ & $\begin{array}{l}\text { Rounded Edge Radius (m) Computed } \\
\text { By ITU-R 526-13 Method }\end{array}$ & Percentage Difference in Radius (\%) \\
\hline 4 & $59,031.42$ & $12,374,693.37$ & $20,862.89$ \\
4.5 & $59,031.42$ & $12,374,693.37$ & $20,862.89$ \\
5 & $59,031.42$ & $12,374,693.37$ & $20,862.89$ \\
5.5 & $59,031.42$ & $12,658,123.28$ & $21,343.03$ \\
6 & $59,031.42$ & $12,658,123.28$ & $21,343.03$ \\
6.5 & $59,031.42$ & $12,658,123.28$ & $21,343.03$ \\
7 & $59,031.42$ & $12,658,123.28$ & $21,343.03$ \\
7.5 & $59,031.42$ & $12,658,123.28$ & $21,343.03$ \\
8 & $59,031.42$ & $12,954,399.00$ & $21,844.92$ \\
\hline
\end{tabular}

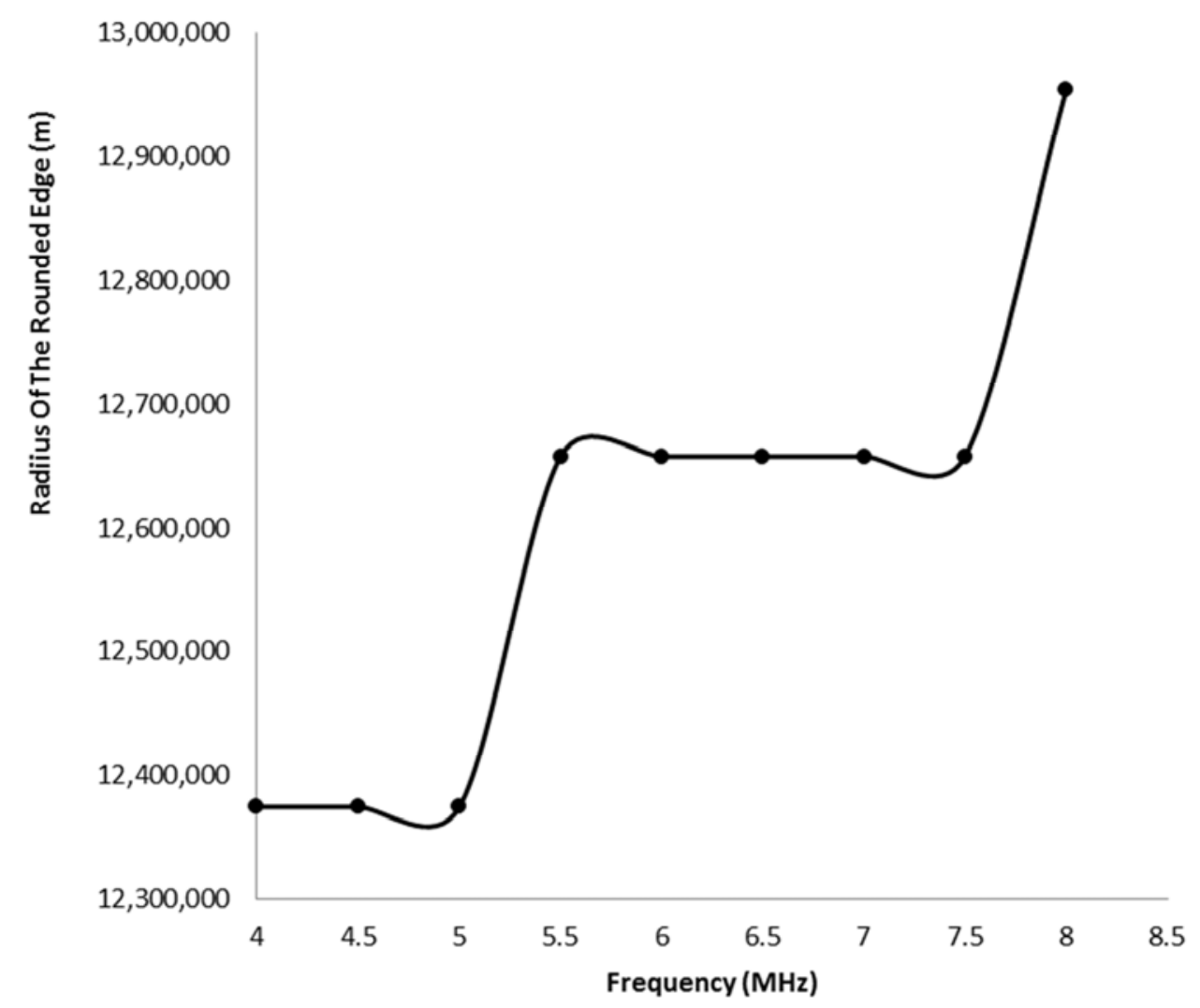

Figure 4. Effect of frequency on the rounded edge radius computed by ITU-R 526-13 method.

Table 4. Comparison of the Effect of Frequency and The Rounded Edge on Diffraction Loss Over The rounded Edge.

\begin{tabular}{|c|c|c|c|}
\hline Frequency (MHz) & $\begin{array}{l}\text { Diffraction Loss (dB) Based on rounded } \\
\text { Edge Radius (m) By Hacking Method }\end{array}$ & $\begin{array}{l}\text { Diffraction Loss (dB) Based on rounded Edge } \\
\text { Radius (m) By ITU-R 526-13 Method }\end{array}$ & $\begin{array}{l}\text { Percentage Difference in } \\
\text { Diffraction Loss }(\%)\end{array}$ \\
\hline 4 & 80.35494 & 334.9047 & 316.78 \\
\hline 4.5 & 82.93134 & 347.6737 & 319.23 \\
\hline 5 & 85.30611 & 359.5115 & 321.44 \\
\hline 5.5 & 87.51339 & 373.149 & 326.39 \\
\hline 6 & 89.57903 & 383.6205 & 328.25 \\
\hline 6.5 & 91.52307 & 393.5154 & 329.96 \\
\hline 7 & 93.36143 & 402.9067 & 331.56 \\
\hline 7.5 & 95.10697 & 411.8535 & 333.04 \\
\hline 8 & 96.77024 & 423.4129 & 337.54 \\
\hline
\end{tabular}

Figure 5. Effect of frequency on the rounded edge radius computed by ITU-R 526-13 method. 


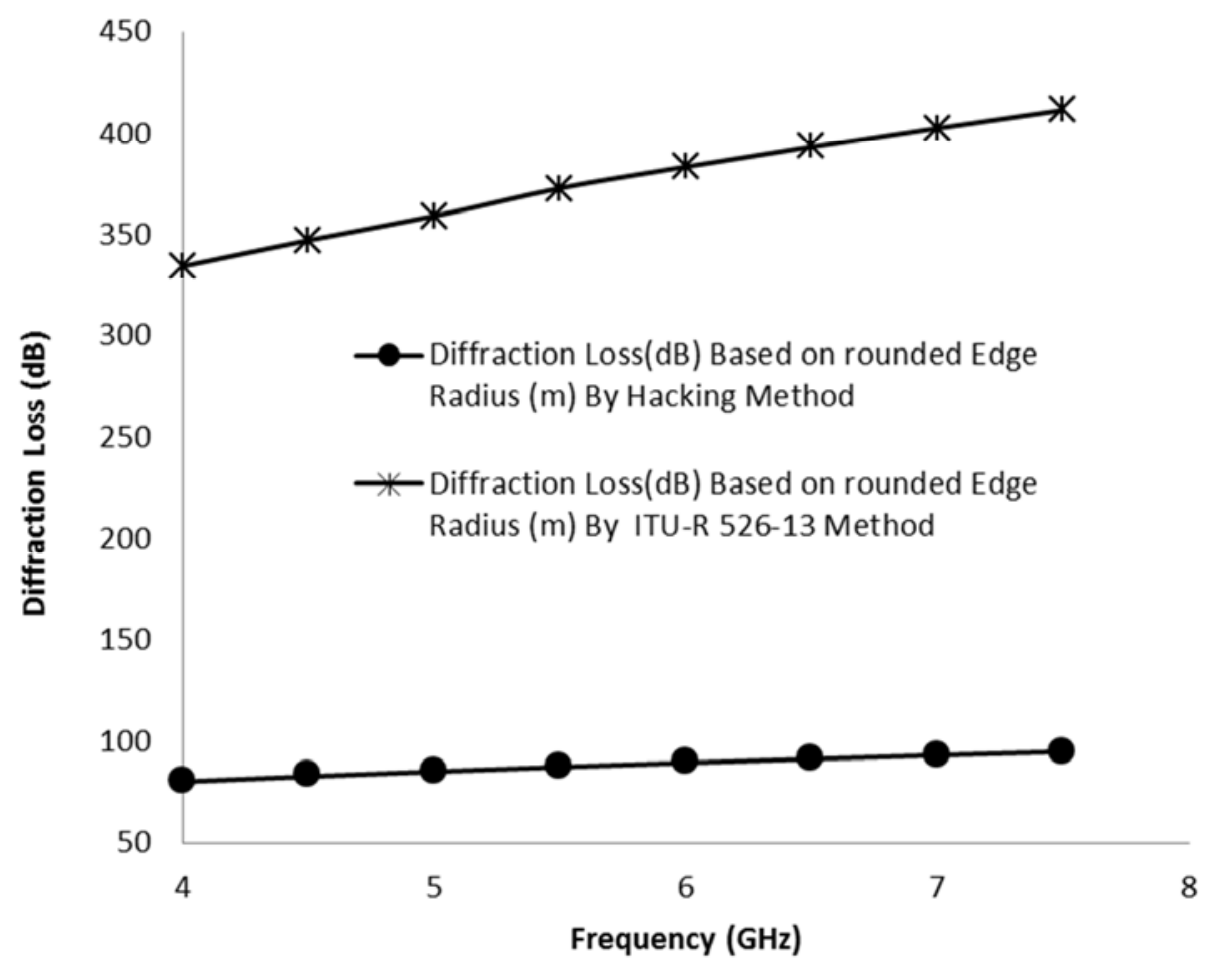

Figure 5. Comparison Of The Effect Of Frequency and The Rounded Edge On Diffraction Loss Over Rounded Edge.

\section{Conclusion}

The effect of the radius of curvature on the diffraction loss of the rounded edge obstruction is presented. The study is conducted for C-band microwave link with a plateau in its path. Two different approaches are used to determine the radius of curvature of the rounded edged fitted to the plateau top. Among the two methods employed, the ITU-R 526-13 method overestimated the radius and also gave very high diffraction loss value for the plateau. In this wise, it can be concluded that the ITU-R 526-13 method is not particularly suitable for estimating the radius of curvature for the rounded edge when applied to a plateau. In addition, a more accurate method is required to estimate the radius of curvature for computing rounded edge diffraction loss.

\section{References}

[1] Seybold, J. S. (2005). Introduction to RF propagation. John Wiley \& Sons.

[2] J. D. Parsons, The Mobile Radio Propagation Channel, 2nd ed., Wiley, West Sussex, 1992, pp. 45-46.

[3] B. McLarnon, VHF/UHF/Microwave Radio Propagation: A Primer for Digital Experimenters, www.tapr.org.

[4] W. C. Y. Lee, Mobile Communication Engineering, Theory and Applications, $2^{\text {nd }}$ ed., McGraw-Hill, New York, 1998, pp. 147149.

[5] N. Blaunstein, Radio Propagation in Cellular Networks, Artech House, Norwood, MA, 2000, pp. 135-137.
[6] Willis, S. L. (2007). Investigation into long-range wireless sensor networks (Doctoral dissertation, James Cook University).

[7] Lazaridis, P. I., Kasampalis, S., Zaharis, Z. D., Cosmas, J. P., Paunovska, L., \& Glover, I. (2015, May). Longley-Rice model precision in case of multiple diffracting obstacles. In URSI Atlantic Conference, Canary Islands.

[8] Östlin, E. (2009). On Radio Wave Propagation Measurements and Modelling for Cellular Mobile Radio Networks.

[9] Kumar, K. A. M. (2011). Significance of Empirical and Physical Propagation Models to Calculate the Excess Path Loss. Journal of Engineering Research and Studies, India.

[10] Malila, B., Falowo, O., \& Ventura, N. (2016, April). Performance analysis of NLOS small cell backhaul using $17 \mathrm{GHz}$ point-to-point prototype radio. In Electrotechnical Conference (MELECON), 2016 18th Mediterranean (pp. 1-6). IEEE.

[11] Jicha, O., Pechac, P., Kvicera, V., \& Grabner, M. (2013). Estimation of the radio refractivity gradient from diffraction loss measurements. IEEE Transactions on Geoscience and Remote Sensing, 51 (1), 12-18.

[12] Silva, F. S., Matos, L. J., Peres, F. A. C., \& Siqueira, G. L. (2013, August). Coverage prediction models fitted to the signal measurements of digital TV in Brazilian cities. In Microwave \& Optoelectronics Conference (IMOC), 2013 SBMO/IEEE MTT-S International (pp. 1-5). IEEE.

[13] Hacking, K. U. H. F. (1968). Propagation over rounded hills. BBC Research Department. Research Report No. RA-21, 30.

[14] International Telecommunication Union, "Recommendation ITU-R P. 526-13: "Propagation by diffraction", Geneva, 2013.

[15] Seybold, J. S. (2005). Introduction to RF propagation. John Wiley \& Sons. 
[16] Barué, G. (2008). Microwave engineering: land \& space radiocommunications (Vol. 9). John Wiley \& Sons.

[17] Jude, O. O., Jimoh, A. J., \& Eunice, A. B. (2016). Software for Fresnel-Kirchoff Single Knife-Edge Diffraction Loss Model. Mathematical and Software Engineering, 2 (2), 76-84.
[18] Rodriguez, I., Nguyen, H. C., Sørensen, T. B., Zhao, Z., Guan, H., \& Mogensen, P. (2016, October). A novel geometrical height gain model for line-of-sight urban micro cells below 6 GHz. In Wireless Communication Systems (ISWCS), 2016 International Symposium on (pp. 393-398). IEEE. 